\title{
ERRATUM
}

\section{Accelerating charging dynamics in subnanometre pores}

Svyatoslav Kondrat, Peng Wu, Rui Qiao and Alexei A. Kornyshev

Nature Materials 13, 387-393 (2014); published online 21 March 2014; corrected after print 25 March 2014.

In the version of this Article originally published, the e-mail address of Svyatoslav Kondrat was misspelt; it should have read 's.kondrat@fz-juelich.de. This error has now been corrected in the online versions of the Article. 